\title{
Mega-Analysis of Gene Expression in Mouse Models of Alzheimer's Disease
}

\author{
Beryl Zhuang, ${ }^{1,2,3}$ B. Ogan Mancarci, ${ }^{1,2,3}{\text { Lilah } \text { Toker, }^{2,3} \text { and }{ }^{-P} \text { Paul Pavlidis }}^{2,3}$
}

https://doi.org/10.1523/ENEURO.0226-19.2019

${ }^{1}$ Graduate Program in Bioinformatics, University of British Columbia, Vancouver V6T1Z4, Canada, ${ }^{2}$ Department of Psychiatry, University of British Columbia, Vancouver V6T1Z4, Canada, and ${ }^{3}$ Michael Smith Laboratories, University of British Columbia, Vancouver V6T1Z4, Canada

\begin{abstract}
While multiple studies have been conducted of gene expression in mouse models of Alzheimer's disease (AD), their findings have not reached a clear consensus and have not accounted for the potentially confounding effects of changes in cellular composition. To help address this gap, we conducted a re-analysis based meta-analysis (mega-analysis) of ten independent studies of hippocampal gene expression in mouse models of AD. We used estimates of cellular composition as covariates in statistical models aimed to identify genes differentially expressed (DE) at either early or late stages of progression. Our analysis revealed changes in gene expression at early phases shared across studies, including dysregulation of genes involved in cholesterol biosynthesis and the complement system. Expression changes at later stages were dominated by cellular compositional effects. Thus, despite the considerable heterogeneity of the mouse models, we identified common patterns that may contribute to our understanding of AD etiology. Our work also highlights the importance of controlling for cellular composition effects in genomics studies of neurodegeneration.
\end{abstract}

Key words: Alzheimer's; bioinformatics; genomics; metaanalysis; transcriptome

\section{Significance Statement}

A molecular understanding of Alzheimer's disease $(A D)$ is important to the development of treatments. Because of the difficulty of studying brain tissue in humans, especially at very early stages of progression, many groups have performed transcriptomic studies of rodent models. Our study identified changes in gene expression that are strikingly consistent across multiple such studies, providing substantial insight into molecular changes present at early stages of these disease models and which may be of importance in the human condition. Our study also demonstrates the importance of accounting for the complex effects of neurodegeneration on brain tissue in data analysis.

\section{Introduction}

There is intense interest in understanding the molecular mechanisms that contribute to Alzheimer's disease (AD), which involve complex interplays of genetic and environmental factors. However, the early changes in the brain before the onset of cognitive impairment in $A D$ are still

Received June 12, 2019; accepted October 24, 2019; First published November 25, 2019.

The authors declare no competing financial interests.

Author contributions: B.Z. and P.P. designed research; B.Z., B.O.M., and L.T. performed research; B.Z. and B.O.M. analyzed data; B.Z., B.O.M., and P.P. wrote the paper. poorly understood (Masters et al., 2015). In AD patients, pathologic changes in the brain often precede the occurrence of clinical symptoms by years (Pennanen et al., 2004; Webster et al., 2014; Wirz et al., 2014). Postmortem brain samples of AD patients represent a late phase of the disorder, and it is challenging to distinguish causes from effects of neurodegeneration. Studying early molecular

This work was supported by the Canadian Institutes of Health Research Joint Program on Neurodegenerative Disease Research, the National Institutes of Health Grant MH111099, the Natural Sciences and Engineering Research Council of Canada, and the University of British Columbia Graduate Program in Bioinformatics. 
changes in mouse models improves our understanding of human disease pathogenesis. Various transgenic mouse models have been developed based on known mutations in familial $A D$ cases and other AD related genes (Webster et al., 2014). Despite the differences in mechanisms, many of these animal models show similar age-dependent progression in decline of cognitive functions that are similar to AD symptoms observed in human (Webster et al., 2014). Identifying genes that are differentially expressed (DE) during early disease phase in the mouse models versus wild type could help understanding early responses to disease initiation, which may be neuroprotective or contribute to disease progression.

Recent expression profiling studies of AD mouse models have reported up-regulation of genes in classical complement cascade and changes of synapse-related genes in early AD disease progression (D'Onofrio et al., 2011; Hong et al., 2013, 2016). Studies of AD mouse models in later phases of progression (Matarin et al., 2015; Saura et al., 2015) often report expression changes of genes related to inflammation and the immune system. However, their findings are not completely concordant. Differences in mouse models can be one of the contributing factors, but differences in experiment design, sample size, microarray platforms, and data processing methods can also influence the results. Since these mouse models develop similar phenotypes, they could share molecular commonalities. A combined analysis of gene expression profiles of mouse models (which we refer to as a megaanalysis, to distinguish it from a meta-analysis, which traditionally refers to pooling of reported findings from studies rather than re-analyzing the raw data) has the potential to identify cross-mouse-model and diseasephase-specific transcriptional changes.

One of the challenges of bulk tissue transcriptome profiling in AD studies is that samples represent a weighted average of cellular composition, and cell counts of different cell types are typically not directly assessed. As a neurodegenerative disorder (ND), AD is characterized by neuronal loss and neuroinflammation (Serrano-Pozo et al., 2011). In AD patients and in some mouse models, loss of pyramidal neurons and other types of neurons in the hippocampus contributes to neuronal loss (Saxena and Caroni, 2011; Serrano-Pozo et al., 2011). Cell-type proportion changes have been identified as one of the driving forces for expression changes in bulk tissues of an $A D$ mouse model (Srinivasan et al., 2016). Therefore, both cellular composition differences and cell type-specific expression differences can contribute to the overall expression changes in bulk tissue samples between disease models and controls, especially at late stages of progres-

Acknowledgements: We thank Weihong Song, Gabriela Cohen Freue, and the Pavlidis lab for discussion. We also thank all the authors who contributed their data to the public repositories, which enabled this study.

Correspondence should be addressed to Paul Pavlidis at paul@msl.ubc.ca. https://doi.org/10.1523/ENEURO.0226-19.2019

Copyright (C) 2019 Zhuang et al.

This is an open-access article distributed under the terms of the Creative Commons Attribution 4.0 International license, which permits unrestricted use, distribution and reproduction in any medium provided that the original work is properly attributed. sion. In contrast to late stages, early effects are expected to be relatively subtle but perhaps more informative about processes leading to pathology.

A recent study examined expression changes of neuronal-specific marker genes in whole brain tissues of human and mouse models of $A D$ and revealed that expression profiles reflect cell population changes (Hokama et al., 2014). To address the cellular composition problem, methods for estimating cell-type proportions from bulk tissue transcriptome profiles have been developed, sometimes referred to as cell-type deconvolution. A commonly used method to deconvolute effects of cell-type proportion changes of bulk tissue is to estimate cell-type proportions by cell type-specific marker genes (Gaujoux and Seoighe, 2013; Shen-Orr and Gaujoux, 2013; Chikina et al., 2015; Mancarci et al., 2017). While not without caveats, accounting for cell type-specific expression changes improves the interpretability of bulk tissue datasets (Mancarci et al., 2017; Toker et al., 2018).

In this study, we re-analyzed publicly available expression data from the hippocampus of multiple AD mouse models, accounting for between-study differences and cell type-specific effects, with the goal of identifying consistent cross-mouse-model transcriptional changes, especially at early phases.

\section{Materials and Methods}

Analyses were implemented in R (Team, 2016) except where noted. The data and scripts used are available at https://github.com/PavlidisLab/AlzMouseModelMeta.

\section{Data pre-processing and quality control}

We retrieved gene expression profiling studies of mouse models of $A D$ with the keyword "Alzheimer" from Gene Expression Omnibus (GEO; RRID:SCR_005012) and ArrayExpress (Barrett et al., 2013; Kolesnikov et al., 2015; RRID:SCR_002964). We further filtered the datasets and selected studies that have at least two biological replicate per condition and contained hippocampal samples. Initially 11 independent studies met the selection criteria. All were conducted on microarray platforms. We downloaded expression data and experimental design meta data from GEO. Probeset and gene annotations of the corresponding Affymetrix, Illumina and Agilent platforms were obtained from Gemma (Zoubarev et al., 2012; RRID: SCR_008007). On further examination, dataset GSE36981 was removed because genotype was confounded with sample batches. This left a final group of 10 datasets. Four major types of $A D$ mouse models are among the 10: amyloid transgenic models, tau transgenic models, knock-out (KO) models and anti-NGF AD11 (categorized as "other"). Individual studies varied in their use of male or female animals, with both sexes included in the aggregated data. For overviews of the datasets, see Tables 1, 2 .

The quality of the raw expression data of Affymetrix arrays was evaluated as described in (Rogic et al., 2016). All hippocampal samples using Affymetrix arrays passed the quality control procedures. Such raw data quality control procedures were not available for Agilent and Illumina arrays. To standardize data processing, Affymetrix and Agilent arrays were robust multi-array aver- 
Table 1. Summary of selected gene expression profiling studies for AD

\begin{tabular}{|c|c|c|c|c|c|c|c|}
\hline Disease & Phase & $\begin{array}{l}\text { Number of } \\
\text { studies }\end{array}$ & $\begin{array}{c}\text { Number of } \\
\text { mouse models }\end{array}$ & $\begin{array}{c}\text { Number of } \\
\text { controls }\end{array}$ & $\begin{array}{c}\text { Number of } \\
\text { disease samples }\end{array}$ & $\begin{array}{c}\text { Total } \\
\text { samples }\end{array}$ & $\begin{array}{l}\text { Total unique } \\
\text { genes }^{\mathrm{c}}\end{array}$ \\
\hline$A D$ & Early & 4 & 9 & 61 & 69 & 116 & 10,853 \\
\hline & Late & 8 & 8 & 50 & 55 & 92 & 10,366 \\
\hline Total & & $10^{a}$ & $12^{b}$ & $84^{c}$ & 124 & 208 & $11,071^{d}$ \\
\hline
\end{tabular}

a Two AD studies are categorized in both early and late disease phases.

b There are shared mouse models between early and late phases, and across studies.

${ }^{c}$ A total of 27 control samples were used as age-matched controls in both early and late phases in dataset GSE64398.

d Total unique genes are counts after removing genes with low expression values.

age (RMA) background corrected by affy (Gautier et al., 2004) and limma (Ritchie et al., 2015) R packages (RRID: SCR_012835, RRID:SCR_010943 respectively) followed by quantile normalization and $\log _{2}$ transformation. Illumina arrays were quantile normalized and $\log _{2}$ transformed. Samples with brain tissues other than hippocampus were discarded after normalization. Samples that were outliers (two standard deviations away from the mean sample-tosample Pearson correlation within a dataset) were removed and the remaining samples were batch-corrected for each dataset by ComBat (Johnson et al., 2007; RRID: SCR_010974) if batch information was available.

The time points when mouse models first develop phenotypes that are similar to the earliest clinical symptoms for diagnosis in $A D$ were used to define early and late phases of AD. The mouse phenotypes of AD mouse models analyzed were based on the behavior data from original publications, or the publications cited in the original paper. Mild cognitive decline is an early diagnostic symptom in AD patients (Webster et al., 2014). Cognitive impairment is often assessed by water maze test in $A D$ mouse models. Therefore, AD mouse samples that did not display impairment in memory and learning measured by water maze tests were categorized as early phase $A D$ samples, while the rest as late phase AD samples. The final dataset constituted data for early time points with controls (116 samples) and late time points with controls (96 samples).

To allow cross-platform comparison, within each dataset, we removed non-specific probes (i.e., probes that mapped to multiple genes), probes that did not map to any genes, and probes that contained missing expression values in one or more samples. When more than one probe mapped to a gene, we retained only the probe with the highest median expression value to represent the mapped gene. Not all the genes are available on all platforms used by the studies; we selected genes that were present in more than at least $2 / 3$ of the platforms as a compromise between maximizing the number of genes in the analysis and the requirement to have multiple measurements to perform a mega-analysis. For each disorder, two integrated datasets were created by combining samples across studies from each disease phase. Within each integrated dataset, gene expression values were quantile normalized to harmonize scales across studies. We then filtered each dataset to remove non-expressed genes. To set the threshold for filtering, we were guided by the expression level of sex-specific genes (Toker et al., 2016). The signal for sex-specific genes in the non-expressing sex (e.g., Y-linked genes in females) can be taken as a rough indicator of background levels. The median expression value of non-expressed sex-specific genes from all samples was 5.2, and thus, we filtered genes with expression value lower than 6 as a more stringent threshold. For the number of genes in each disease phase after gene filtering, see Table 1.

Most of AD mouse models analyzed in this project were transgenic mouse models with transgenes under the control of murine Thy 1 tissue-specific regulatory elements. The microarray probesets mapped to these transgenes

Table 2. Details of analyzed AD mouse model studies

\begin{tabular}{|c|c|c|c|c|c|c|c|}
\hline $\begin{array}{l}\text { Model } \\
\text { types }\end{array}$ & Mouse model(s) & Study (dataset) & Phase(s) & Samples & Platform & Genes & $\mathrm{M} / \mathrm{F}$ \\
\hline \multirow{4}{*}{ Amyloid } & & GSE1556 (Stein et al., 2004) & Late & $4(2 / 2)$ & GPL81 & 8237 & $0 / 4$ \\
\hline & $5 x F A D$ & GSE52022 (Noh et al., 2014) & Late & $4(2 / 2)$ & GPL1261 & 18118 & $0 / 4$ \\
\hline & & GSE50521 (Paesler et al., 2015) & Late & $12(6 / 6)$ & GPL6096 & 16743 & $8 / 4$ \\
\hline & $\mathrm{J} 20$ & GSE14499 (Nagahara et al., 2009) & Late & $6(2 / 4)$ & GPL1261 & 18118 & $2 / 4$ \\
\hline TAU & rTg4510 & GSE53480 (Polito et al., 2014) & Late & $8(4 / 4)$ & GPL1261 & 18118 & $5 / 3$ \\
\hline $\mathrm{KO}$ & $\begin{array}{l}\text { Aplp2 KO, App KO, } \\
\text { App/Aplp2 } \\
\text { double-conditional } \\
\text { KO (NdC-KO) }\end{array}$ & GSE48622 (Li et al., 2010) & Early & $16(4 / 12)$ & GPL1261 & 18118 & $16 / 0$ \\
\hline Other & Anti-NGF AD11 (AD11) & GSE63617 (D’Onofrio et al., 2011) & Early, late & $30(15 / 15)$ & $\begin{array}{l}\text { GPL7042, } \\
\text { GPL7202 }\end{array}$ & 19459 & $0 / 30$ \\
\hline
\end{tabular}

Number of control and case samples are shown in parentheses (control/case). Genes refer to the number of unique genes mapped. M/F: number of male and female samples. Amyloid: amyloid transgenic models; TAU: TAU transgenic models. 
and the endogenous copy, which artificially increased the measured expression of Thy1. Therefore, Thy1 was removed from the mega-analysis in AD mouse models.

\section{Estimation of cell-type proportion changes}

Cell-type proportions of three glial cell types (microglia, astrocytes, oligodendrocytes) and three neuronal cell types (pyramidal cells, dentate granule cells, GABAergic cells) were estimated by marker gene profiles (MGPs) using pre-selected markers specific to the murine hippocampus (Mancarci et al., 2017). Expression of the marker genes were first corrected for between-study variation for each disease phase and then used as input for MGP estimation. For presentation MGPs were normalized to a range between 0 and 1 , where the sample with the highest profile was assigned to 1 and the lowest was assigned to 0 . Wilcoxon rank-sum test and computation of false discovery rates (FDRs) using the Benjamini-Hochberg procedure (Benjamini and Hochberg, 1995) were applied to test whether the profiles were significantly different between disease mouse models and controls for each cell type.

\section{Fitting linear mixed-effects models (LMMs) and jackknife procedure to rank genes}

LMMs allow modeling multiple sources of variation, such as mouse model-specific effects, laboratory effects, and difference between disease models and controls (Demirkale et al., 2010). For each disease phase group, two LMMs were fitted for each gene using the "Imer" function in $\mathrm{R}$ package Ime4 version 1.1-12 (RRID: SCR_015654), via maximum likelihood estimation [1mer( REML $=$ F ) ; Bates et al., 2015]. The first LMM corrected for between-study variations without correction for the MGPs; the second LMM corrected for both betweenstudy variations and MGPs. The $p$ value for the significance of the fixed effect of disease state (i.e., disease and normal states) was obtained by the "anova" function in R package stats version 3.3.1 (Chambers and Hastie, 1991). Ranking of upregulated or downregulated genes was based on $p$ values in ascending order and the direction of expression changes between mouse models and controls. FDR estimated by the Benjamini-Hochberg procedure was computed for each gene. Significantly DE genes had FDR $<0.05$. A jackknife procedure was applied to yield more robust gene rankings.

\section{Functional enrichment analysis}

Functional enrichment analysis was performed using the threshold-free precision-recall algorithm in ErmineJ version 3.0.2 (Gillis et al., 2010; RRID:SCR_006450) to determine enrichment of Gene Ontology (GO) terms for the ranked list of genes from the jackknife procedure. We used the multifunctionality-adjusted enrichment rankings provided by ErmineJ to reduce the distorting effect of highly annotated genes (Ballouz et al., 2016).

\section{Results}

We reanalyzed a total of 208 gene expression profiles from $10 \mathrm{AD}$ mouse model datasets (Tables 1, 2). With the goal of identifying shared transcriptional alterations among different mouse models in the early and late phases, two separate mega-analyses were conducted: analysis of samples from "early" time points (before most pathologic effects), and a later time point. An important feature of our analysis is the use of MGPs. Used directly, MGPs can be used as an estimate of relative cellular proportions (Mancarci et al., 2017). We also used MGPs as covariates in our linear models to help isolate changes due to regulation as opposed to changes in cellular makeup of the tissue.

\section{Estimation of cell-type proportion changes}

The results of MGP estimation were largely consistent with the expected cell-type changes in the early and late phase mouse samples. Relative to controls, mouse model samples in the early phase were predicted to show minimal or small cellular composition changes while late phase samples would show more substantial changes. In agreement, with this hypothesis, during the early phase, MGP analysis indicated no significant changes in neurons and glial cells (Fig. 1A,B). During the late phase, $A D$ mouse models had estimated reduced dentate granule cells and pyramidal cells (Fig. 1A), and increased astrocytes and microglia (Fig. 1B).

\section{Mega-analysis of gene expression in AD mouse models}

Using a mixed effect modeling approach with MGPs as covariates (see Materials and Methods), we identified expression changes associated with early and late phases of the AD mouse models. As expected, early changes were subtle. The mega-analysis of 116 gene expression profiles of mouse models and controls in the early phase revealed small but consistent expression changes (Fig. 2). Only four upregulated and three downregulated genes of the top genes were significant at an FDR of 0.05. A few of the top genes were functionally related based on the enrichment analysis. Three of the top 40 upregulated genes (Sqle, Msmo1, and Nsdh/ ranked 2, 3, and 7; FDR $<0.05$ except Nsdh1, which met an FDR of 0.08) are involved in cholesterol biosynthesis. C1qa, C1qb, and C1qc, which are involved in the classical complement cascade, were among the top 40 upregulated gene in the early phase (ranks 28, 12, and 29, meeting an FDR of 0.12).

In contrast to the subtle changes in the early phase, the expression change signals in the late phase were stronger (Fig. 3). The analysis also detected down-regulation of a known AD risk gene, Trem2, which had also been reported in the original publications of two studies analyzed. Several GO terms were found significantly enriched in the top downregulated genes, including "neuronal cell body" and "regulation of neurogenesis."

We note that using MGPs as covariates have important effects on the results, which we documented by comparing the results of fitting models in which MGPs are not included. First, after MGP correction, as expected the marker genes included in the MGPs themselves are down-ranked, especially in the late phase. The number of significant DE genes (FDR < 0.05) in the late phase was greatly reduced after MGP correction:13.64\% of the total genes were DE genes before correction and dropped to 

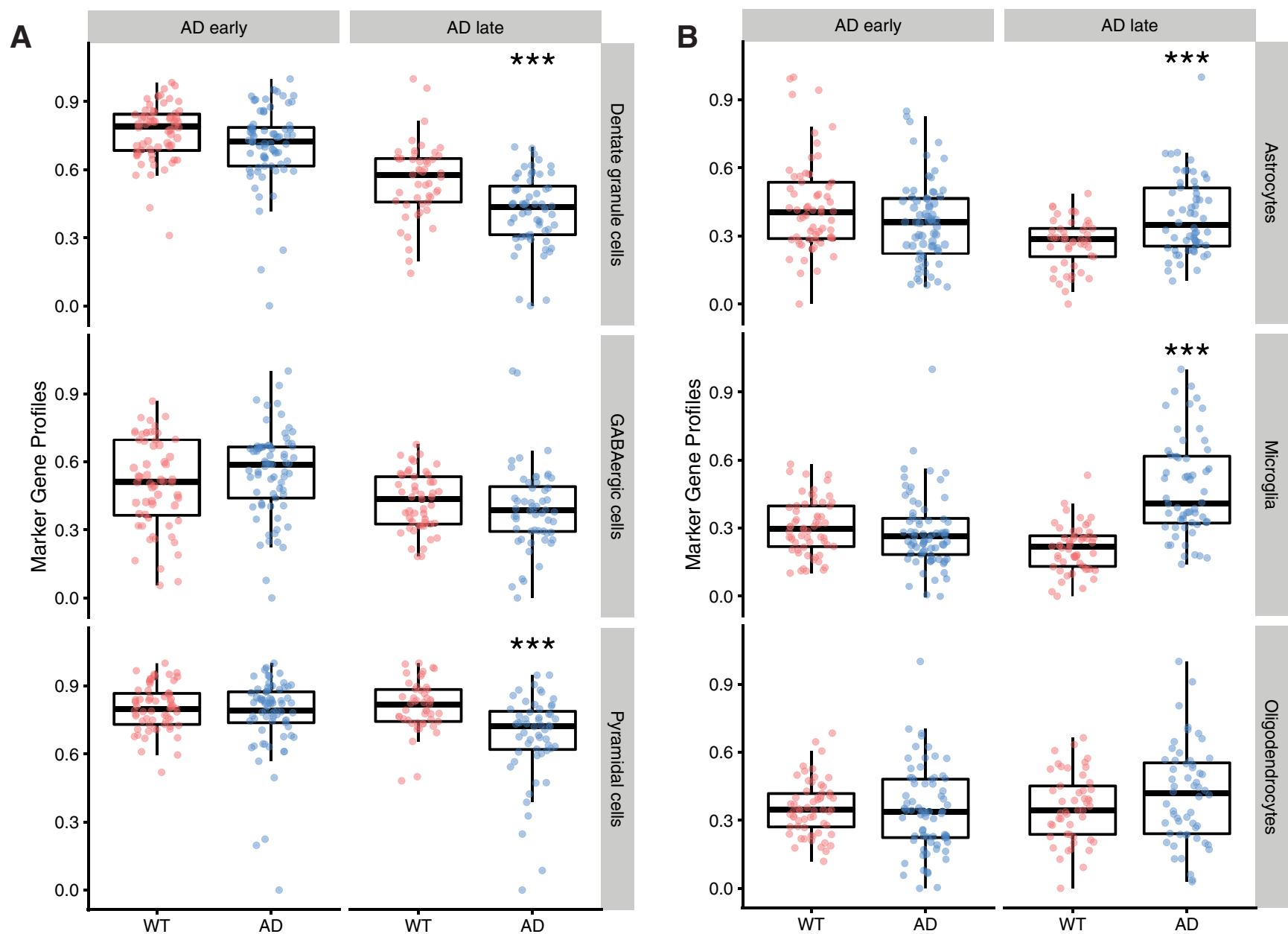

Figure 1. MGPs in AD mouse models. Each row of plots represents MGPs for one cell type as labeled at right. Each point represents one sample. Boxplots representing the interquartile range and the sample means are also shown. The vertical axis is normalized to the range 0 and 1. $* * *$ FDR $<0.01$. $\boldsymbol{A}$, Neurons $\boldsymbol{B}$, Glial cells.

$0.22 \%$ after correction. We anticipated that the additional parameters included in the models would reduce power, so the fact that fewer genes met the FDR threshold was not in itself surprising. However, the gene rankings changed considerably, especially in the late phase. The Spearman correlation coefficients of before and after correction in the late phase is 0.38 , compared to 0.80 in the early phase. Thus, MGP correction affected both the number of DE genes and the gene rankings, which indicated that cellular composition changes (as measured by MGPs) contributed to bulk tissue gene expression changes, especially in the late disease phase.

The gene rankings of early and late $A D$ were quite different, indicating phase-specific effects. The Spearman correlation coefficient for gene ranking of all the genes between early and late phase was 0.24 before MGPs correction and 0.25 after. There were very few genes in common among the top 100 dysregulated genes across the phases. Specifically, only the top genes involved in cholesterol biosynthesis (Sqle, Msmo1, Nsdhl) were dysregulated in concordance in both phases (FDR $<0.05$ ) for both before and after MGPs correction.

\section{Discussion}

We identified consistent transcriptomic alterations across mouse models of AD. By categorizing samples into early and late disease phases, gene expression changes specific to the disease phase were revealed. This revealed subtle but biologically interpretable changes shared across mouse models in the early phase, which may reflect reveal early disease mechanisms. Changes in the late phase were stronger, and as expected were more associated with changes in cellular populations. The topranked genes in the early phase were not always affected in the late phase, and vice versa, indicating phasespecific expression changes.

\section{Consistent transcriptomic alterations were identified across different mouse models}

Candidate genes identified in the original published reports were often inconsistent, though some genes are reported in more than one study. This may be due to differences in analysis methods and thresholds used, but doing a mega-analysis has the advantage of facilitating detection of weaker signals. Thus our mega-analysis was able to capture consistent signals that were not reported 
WT

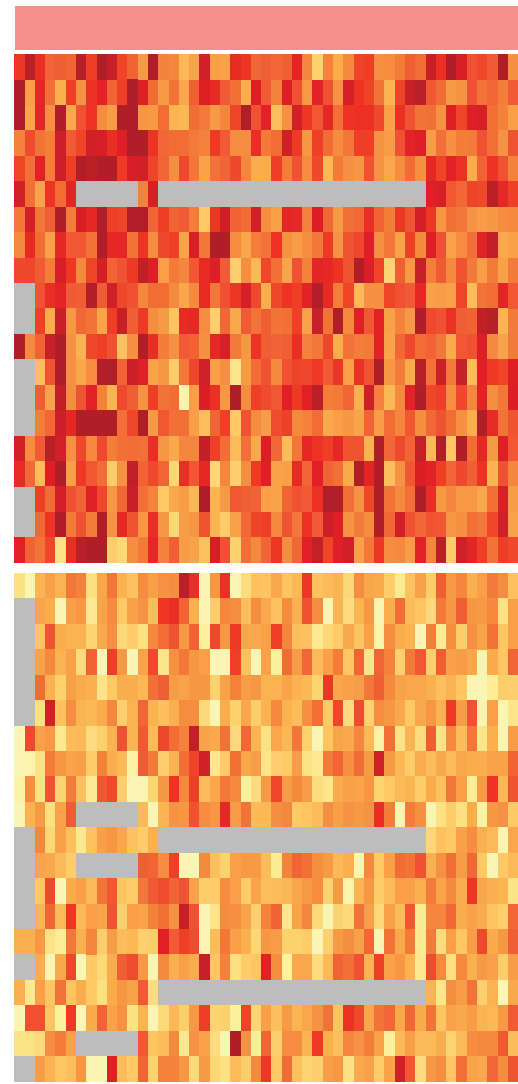

AD models

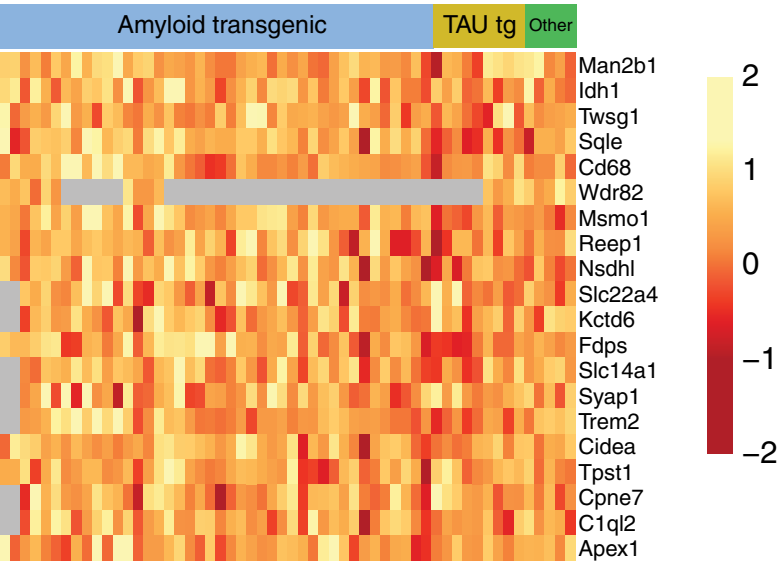

2

0

$-1$

$-2$

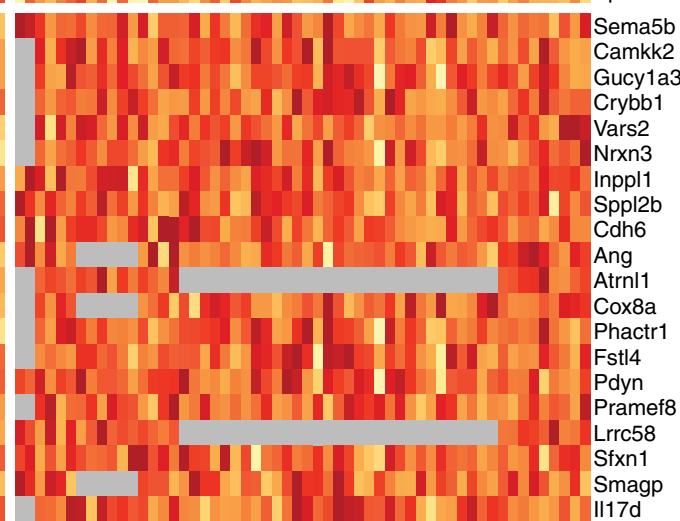

Figure 2. Top 20 upregulated and downregulated genes for late phase. Expression values are corrected for studies and MGPs. Each column is a brain sample, each row is a gene. For display purposes, each row is $z$ score transformed. Gray cells represent missing values. A gap separates the upregulated genes (top) and the downregulated genes (bottom).

in the original source studies. For example, in the late $A D$ phase, two out of seven studies reported the $A D$ risk gene, Trem2, in their hit lists. However, we observed consistent up-regulation of Trem2 in other studies (Fig. $4 A)$. Similarly, we identified genes that had not been reported in any of the original studies, included a top upregulated gene, Msmo1, in the late AD phase (Fig. 4).

\section{Applying cell population proportion correction revealed transcriptional changes of cell type-specific regulatory events}

We used MGPs to adjust the expression profiles for the effects of cellular proportion changes (Mancarci et al., 2017). We note that MGP analysis should interpreted as reflecting cellular proportions with caution, as it is an indirect measure. A more conservative interpretation of an MGP change is a cell type-specific change in expression. However, given the known neurodegeneration in these mouse models, changes in cellular proportions are the likely cause in MGP shifts in our study (Serrano-Pozo et al., 2011). Indeed, the cell-type proportion changes as estimated by MGPs in AD mouse models were consistent with previous mouse and human studies (Schmitz et al., 2004; Serrano-Pozo et al., 2011; Hokama et al., 2014), and indicated neuronal loss and gliosis in the hippocampus in the late phase (Fig. 1A,B). The lack of significant proportion changes of neurons and glial cells before the occurrence of cognitive impairment in $A D$ mouse models (Fig. $1 A, B$ ) also agreed with a report that cognitive impairment, neuronal loss and gliosis occur concurrently in the hippocampus of $A D$ mouse model J20 (Wright et al., 2013). Therefore, we interpret the MGP shifts as cellular proportion changes while keeping the indirect nature of the measure in mind.

The changes of gene rankings, and decreased DE signal after MGPs correction, implies that the gene expression changes, especially in the late disease phase, were substantially driven by changes in cell-type proportions. Adjusting MGPs can reveal cell type-specific transcriptional changes. Some of the markers remained topranked of the same direction of regulation even after correction, such as top-up-ranked microglia markers Cd68 and Tyrobp, and astrocyte marker Slc14a1 in the late $A D$ phase remained top-up-ranked after correction. Because the dysregulation of these marker genes cannot be fully explained by the MGP changes, they may indicate changes at transcriptional regulation level within microglia that contribute to disease pathophysiology. However, because the changes in composition are confounded with the experimental condition, it is also possible that some residual effects of cellular proportion remain even after including MGPs as covariates. Ultimately resolution of cell type-specific changes in gene expression in these mod- 
WT
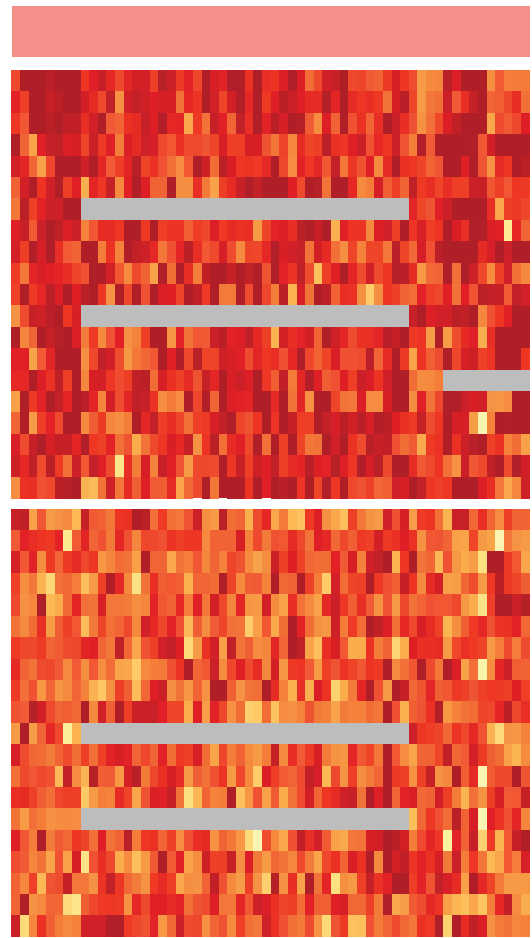

AD models

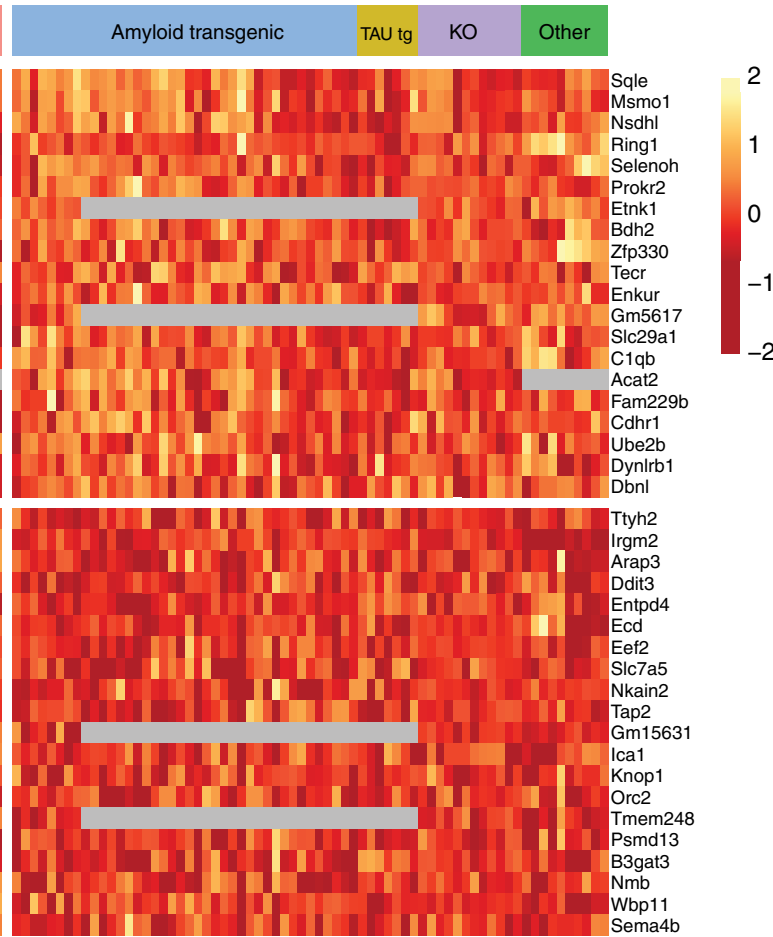

Figure 3. Top 20 upregulated and downregulated genes for early phase. As in Figure 2, but for the early phase.

els, especially at late stages, will require cell type-specific transcriptomic studies.

\section{Shared gene expression changes revealed upregulation of genes in cholesterol biosynthesis and classical complement cascade in AD mouse models}

Our mega-analysis of the early phase revealed upregulation of genes in cholesterol biosynthesis and classical complement cascade. The changes were subtle in the early phase compared to the late phase. However, the results had some biological coherence as suggested by the GO enrichment analysis. Thus, the enrichment of genes involved in cholesterol biosynthesis in both early and late phases, even after MGP correction, suggested chronic dysregulation in cholesterol biosynthesis. Upregulation of genes in cholesterol biosynthesis has been also observed in an AD amyloid transgenic mouse model, APP23 (Tseveleki et al., 2010). Many lines of evidence have linked cholesterol to $A D$, and to $A \beta$ production in particular (Puglielli et al., 2003; Wood et al., 2014), though the interpretation and implications are still unclear. In mouse and cell culture studies, decreased brain cholesterol levels can reduce $A \beta$ abundance (Di Paolo and Kim, 2011; Wood et al., 2014). Cleavage of APP by $\beta$-secretase and $\gamma$-secretase (the amyloidogenic pathway) mainly occurs in lipid rafts of the plasma membrane, whereas $\alpha$-secretase of the non-amyloidogenic pathway tend to localize at the non-lipid-raft sites (Kim et al., 2016). Lipid rafts have high concentration of cholesterol (Kim et al., 2016). Increased level of cholesterol enhances localization of APP, $\beta$-secretase and $\gamma$-secretase to the lipid rafts, and subsequently promotes $A \beta$ production (Marquer et al., 2011; Kim et al., 2016). However, the mechanisms that initiate the increased gene expression of cholesterol biosynthesis genes in mouse models are not known. The majority of samples analyzed here were amyloid transgenic models, which express human transgenes (APP, PSEN1, PSEN2) with known AD associated mutations that promote APP processing through the amyloidogenic pathway. Expression of these transgenes could play a role in promoting the expression of cholesterol biosynthesis genes, which lead to increased cholesterol level that can accelerate $A \beta$ production. The most established AD risk factor is apolipoprotein $\mathrm{E}$ (ApoE, encoded by $A P O E$ ), which mediates cholesterol metabolism in the brain and is found in $A \beta$ plaques and neurofibrillary tangles (Yang and Song, 2013; Ries and Sastre, 2016). It has been shown that the high-risk isoform APOE4 has lower efficiency in transporting cholesterol from astrocytes to neurons compared to the neutral isoform APOE3, and lead to synaptic dysfunctions $(\mathrm{Bu}, 2009)$. Our results suggest cholesterol may play a role in $\mathrm{AD}$-like process initiation and progression in mouse models.

Complement pathway genes, C1qa, C1qb, and C1qc, were among the top-ranked upregulated genes in the early phase, suggested up-regulation of genes in the $\mathrm{C} 1 \mathrm{q}$ pathway in early disease progression. The up-regulation of $\mathrm{C} 1 \mathrm{q}$ pathway, which initiates classical complement cascade, has been linked to early synaptic loss before $A \beta$ accumulation, and is a response to injury in mouse model, and could be neuroprotective against misfolded proteins (Benoit et al., 2013; Hong et al., 2016). C1q pathway genes were still differentially upregulated in the late 

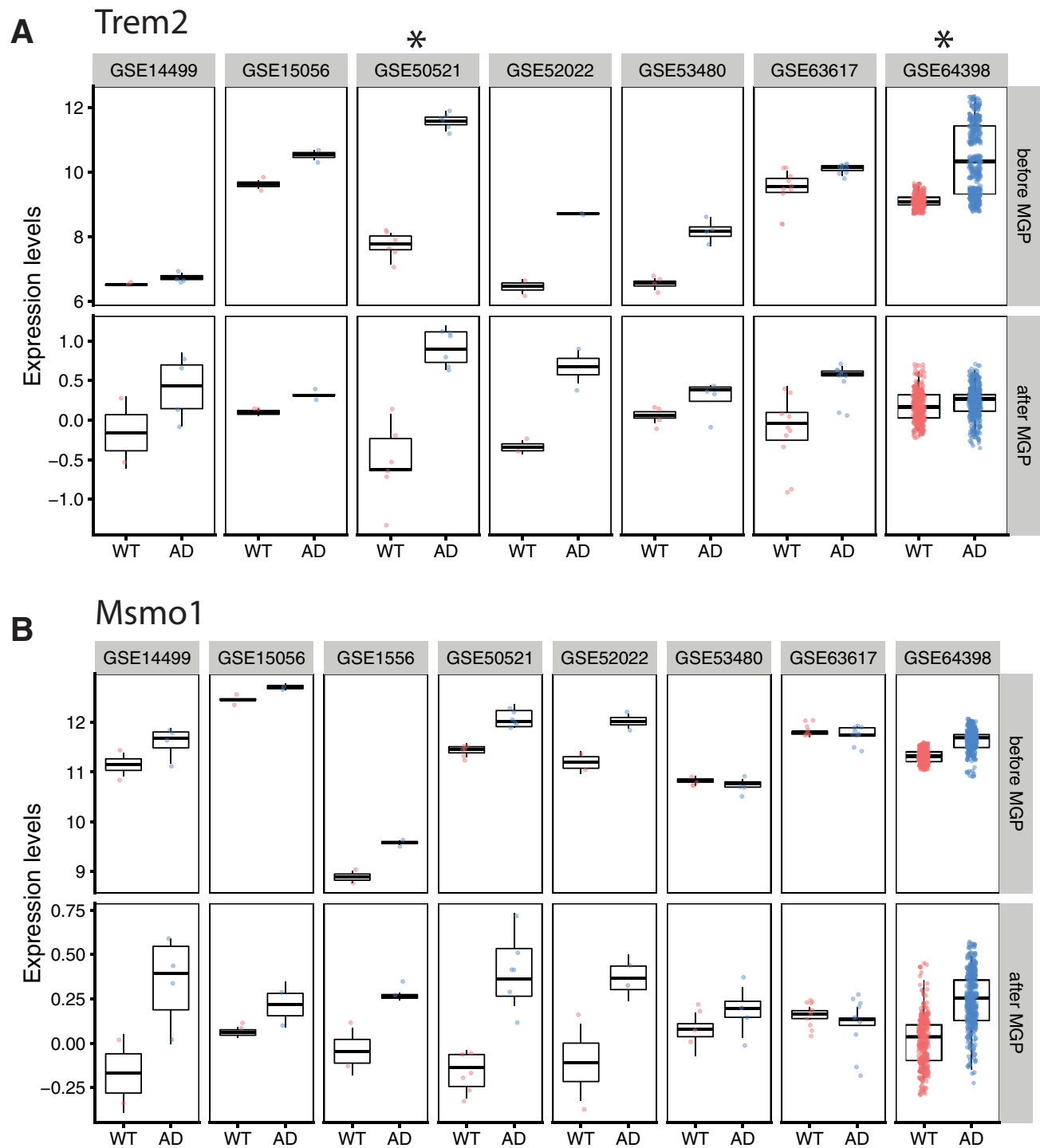

Figure 4. Examples of gene expression patterns identified. Each point represents one sample. Control samples (WT) are marked red and AD mouse model samples are blue. "After MGP" reflects analyses in which MGPs were included as covariates in the model; this correction rescales the expression levels to arbitrary units. Studies (datasets) that are marked with an asterisk have reported the gene as top hit in the original publications. A, Expression of Trem2 in the late AD phase before (top row) and after (bottom row) MGP correction. B, Expressions of Msmo1 in the late AD phase before (top) and after MGP correction (bottom).

phase, however, they were no longer among the topranked genes after MGP correction. This suggests that cellular composition changes are more responsible for RNA levels changes of C1q genes in the late phase.

In the late phase, the GO term regulation of neurogenesis was enriched in the downregulated genes. The result was consistent with previous studies (Noh et al., 2014; Matarin et al., 2015), reflecting neuronal dysfunction in the AD mouse brain, though as noted above this could also potentially reflect imperfect correction for proportion effects. Interestingly, GO terms related to inflammation and the immune system were not enriched in upregulated genes. These results are contrary to other studies, which report up-regulations of genes related to inflammation and immune system (Matarin et al., 2015; Saura et al., 2015). This seeming discrepancy can be explained as a combination of effects of cell-type proportion considerations and gene function annotations. Without MGPs correction, our results did indicate up-regulation of genes in the immune response pathways. However, closer inspection revealed that some of the microglia markers are annotated with GO terms "immune response" and "inflammatory response." This further complicates separating cell-type proportion changes regulatory effects. On the other hand, the dysregulation of a few astrocytes and microglia markers could not be fully explained by the cell-type population changes and remained top-ranked dysregulated genes after MGPs correction. For example, Cd68, a marker for microglial activation and is correlated with $A \beta 42$ load (Zotova et al., 2011), was upregulated in the late phase. Another microglia marker, TYRO protein tyrosine kinase-binding protein gene (Tyrobp) and its re- 
ceptor Trem2 were among the top upregulated genes. A previous study reported that TYROBP binds to TREM2 and promotes microglial activation (Kobayashi et al., 2016). These results may provide some evidence of microglial activation at the cell type-specific level in $A D$ mouse models.

In conclusion, our mega-analysis of gene expression in mouse models revealed consistent and disease phasespecific transcriptional changes and cell type-specific regulatory events, despite the considerable heterogeneity of the mouse models of $A D$. The identification of shared gene expression changes in the early phase increases our understanding of disease initiation and progression. Prioritized top-ranked genes in the early phase can be candidate genes to study mechanisms in disease initiation and worthy of further investigation.

\section{References}

Ballouz S, Pavlidis P, Gillis J (2016) Using predictive specificity to determine when gene set analysis is biologically meaningful. Nucleic Acids Res 45:e20.

Barrett T, Wilhite SE, Ledoux P, Evangelista C, Kim IF, Tomashevsky M, Marshall KA, Phillippy KH, Sherman PM, Holko M, Yefanov A, Lee H, Zhang N, Robertson CL, Serova N, Davis S, Soboleva A (2013) NCBI GEO: archive for functional genomics data setsupdate. Nucleic Acids Res 41:D991-D995.

Bates D, Mächler M, Bolker B, Walker S (2015) Fitting linear mixedeffects models using Ime4. J Stat Softw 67:1-48.

Benjamini Y, Hochberg Y (1995) Controlling the false discovery rate: a practical and powerful approach to multiple testing. J R Stat Soc Ser B Methodol 57:289-300.

Benoit ME, Hernandez MX, Dinh ML, Benavente F, Vasquez O, Tenner AJ (2013) C1q-induced LRP1B and GPR6 proteins expressed early in Alzheimer disease mouse models, are essential for the $\mathrm{C} 1 \mathrm{q}$-mediated protection against amyloid- $\beta$ neurotoxicity. $\mathrm{J}$ Biol Chem 288:654-665.

Bu G (2009) Apolipoprotein E and its receptors in Alzheimer's disease: pathways, pathogenesis and therapy. Nat Rev Neurosci 10:333-344.

Chambers JM, Hastie TJ (1991) Statistical models in S. Boca Raton, FL: Chapman and Hall/CRC.

Chikina M, Zaslavsky E, Sealfon SC (2015) CellCODE: a robust latent variable approach to differential expression analysis for heterogeneous cell populations. Bioinformatics 31:1584-1591.

Cummings DM, Liu W, Portelius E, Bayram S, Yasvoina M, Ho S-H, Smits H, Ali SS, Steinberg R, Pegasiou C-M, James OT, Matarin M, Richardson JC, Zetterberg H, Blennow K, Hardy JA, Salih DA, Edwards FA (2015) First effects of rising amyloid- $\beta$ in transgenic mouse brain: synaptic transmission and gene expression. Brain $\mathrm{J}$ Neurol 138:1992-2004.

Demirkale CY, Nettleton D, Maiti T (2010) Linear mixed model selection for false discovery rate control in microarray data analysis. Biometrics 66:621-629.

Di Paolo G, Kim T-W (2011) Linking lipids to Alzheimer's disease: cholesterol and beyond. Nat Rev Neurosci 12:284-296.

D'Onofrio M, Arisi I, Brandi R, Di Mambro A, Felsani A, Capsoni S, Cattaneo A (2011) Early inflammation and immune response $m R-$ NAs in the brain of AD11 anti-NGF mice. Neurobiol Aging 32:10071022.

Gaujoux R, Seoighe C (2013) CellMix: a comprehensive toolbox for gene expression deconvolution. Bioinformatics 29:2211-2212.

Gautier L, Cope L, Bolstad BM, Irizarry RA (2004) affy-analysis of Affymetrix GeneChip data at the probe level. Bioinforma Oxf Engl 20:307-315

Gillis J, Mistry M, Pavlidis P (2010) Gene function analysis in complex data sets using ErmineJ. Nat Protoc 5:1148-1159.
Hokama M, Oka S, Leon J, Ninomiya T, Honda H, Sasaki K, Iwaki T, Ohara T, Sasaki T, LaFerla FM, Kiyohara Y, Nakabeppu Y (2014) Altered expression of diabetes-related genes in Alzheimer's disease brains: the Hisayama study. Cereb Cortex 24:2476-2488.

Hong H, Hong Q, Liu J, Tong W, Shi L (2013) Estimating relative noise to signal in DNA microarray data. Int $\mathrm{J}$ Bioinforma Res Appl 9:433-448.

Hong S, Beja-Glasser VF, Nfonoyim BM, Frouin A, Li S, Ramakrishnan S, Merry KM, Shi Q, Rosenthal A, Barres BA, Lemere CA, Selkoe DJ, Stevens B (2016) Complement and microglia mediate early synapse loss in Alzheimer mouse models. Science 352:712716.

Johnson WE, Li C, Rabinovic A (2007) Adjusting batch effects in microarray expression data using empirical Bayes methods. Biostatistics 8:118-127.

Kim Y, Kim C, Jang HY, Mook-Jung I (2016) Inhibition of cholesterol biosynthesis reduces $\gamma$-secretase activity and amyloid- $\beta$ generation. J Alzheimers Dis 51:1057-1068.

Kleiman RJ, Lanz TA, Finley JE, Bove SE, Majchrzak MJ, Becker SL, Carvajal-Gonzales S, Kuhn AM, Wood KM, Mariga A, Nelson FR, Verhoest PR, Seymour PA, Stephenson DT (2010) Dendritic spine density deficits in the hippocampal CA1 region of young Tg2576 mice are ameliorated with the PDE9A inhibitor PF-04447943. Alzheimers Dement 6:S563-S564.

Kobayashi M, Konishi H, Sayo A, Takai T, Kiyama H (2016) TREM2/ DAP12 signal elicits proinflammatory response in microglia and exacerbates neuropathic pain. J Neurosci 36:11138-11150.

Kolesnikov N, Hastings E, Keays M, Melnichuk O, Tang YA, Williams E, Dylag M, Kurbatova N, Brandizi M, Burdett T, Megy K, Pilicheva E, Rustici G, Tikhonov A, Parkinson H, Petryszak R, Sarkans U, Brazma A (2015) ArrayExpress update-simplifying data submissions. Nucleic Acids Res 43:D1113-D1116.

Li H, Wang B, Wang Z, Guo Q, Tabuchi K, Hammer RE, Südhof TC, Zheng $H$ (2010) Soluble amyloid precursor protein (APP) regulates transthyretin and Klotho gene expression without rescuing the essential function of APP. Proc Natl Acad Sci USA 107:1736217367.

Mancarci BO, Toker L, Tripathy SJ, Li B, Rocco B, Sibille E, Pavlidis $P$ (2017) Cross-laboratory analysis of brain cell type transcriptomes with applications to interpretation of bulk tissue data. eNeuro 4:ENEURO.0212-17.2017.

Marquer C, Devauges V, Cossec JC, Liot G, Lécart S, Saudou F, Duyckaerts C, Lévêque-Fort S, Potier MC (2011) Local cholesterol increase triggers amyloid precursor protein-Bace1 clustering in lipid rafts and rapid endocytosis. FASEB J 25:1295-1305.

Masters CL, Bateman R, Blennow K, Rowe CC, Sperling RA, Cummings JL (2015) Alzheimer's disease. Nat Rev Dis Primer 1:15056.

Matarin M, Salih DA, Yasvoina M, Cummings DM, Guelfi S, Liu W, Nahaboo Solim MA, Moens TG, Paublete RM, Ali SS, Perona M, Desai R, Smith KJ, Latcham J, Fulleylove M, Richardson JC, Hardy $\mathrm{J}$, Edwards FA (2015) A genome-wide gene-expression analysis and database in transgenic mice during development of amyloid or tau pathology. Cell Rep 10:633-644.

Nagahara AH, Merrill DA, Coppola G, Tsukada S, Schroeder BE, Shaked GM, Wang L, Blesch A, Kim A, Conner JM, Rockenstein E, Chao MV, Koo EH, Geschwind D, Masliah E, Chiba AA, Tuszynski $\mathrm{MH}$ (2009) Neuroprotective effects of brain-derived neurotrophic factor in rodent and primate models of Alzheimer's disease. Nat Med 15:331-337.

Noh H, Park C, Park S, Lee YS, Cho SY, Seo H (2014) Prediction of miRNA-mRNA associations in Alzheimer's disease mice using network topology. BMC Genomics 15:644.

Paesler K, Xie K, Hettich MM, Siwek ME, Ryan DP, Schröder S, Papazoglou A, Broich K, Müller R, Trog A, Garthe A, Kempermann G, Weiergräber M, Ehninger D (2015) Limited effects of an elF2 $\alpha$ S51A allele on neurological impairments in the 5xFAD mouse model of Alzheimer's disease. Neural Plast 2015:825157.

Pennanen C, Kivipelto M, Tuomainen S, Hartikainen P, Hänninen T, Laakso MP, Hallikainen M, Vanhanen M, Nissinen A, Helkala E-L, Vainio P, Vanninen R, Partanen K, Soininen H (2004) Hippocampus 
and entorhinal cortex in mild cognitive impairment and early AD. Neurobiol Aging 25:303-310.

Pereson S, Wils H, Kleinberger G, McGowan E, Vandewoestyne M, Van Broeck B, Joris G, Cuijt I, Deforce D, Hutton M, Van Broeckhoven C, Kumar-Singh S (2009) Progranulin expression correlates with dense-core amyloid plaque burden in Alzheimer disease mouse models. J Pathol 219:173-181.

Polito VA, Li H, Martini-Stoica H, Wang B, Yang L, Xu Y, Swartzlander DB, Palmieri M, di Ronza A, Lee VY, Sardiello M, Ballabio A, Zheng H (2014) Selective clearance of aberrant tau proteins and rescue of neurotoxicity by transcription factor EB. EMBO Mol Med 6:1142-1160.

Puglielli L, Tanzi RE, Kovacs DM (2003) Alzheimer's disease: the cholesterol connection. Nat Neurosci 6:345-351.

Ries M, Sastre M (2016) Mechanisms of $A \beta$ clearance and degradation by glial cells. Front Aging Neurosci 8:160.

Ritchie ME, Phipson B, Wu D, Hu Y, Law CW, Shi W, Smyth GK (2015) limma powers differential expression analyses for RNAsequencing and microarray studies. Nucleic Acids Res 43:e47.

Rogic S, Wong A, Pavlidis P (2016) Meta-Analysis of Gene Expression Patterns in Animal Models of Prenatal Alcohol Exposure Suggests Role for Protein Synthesis Inhibition and Chromatin Remodeling. Alcohol Clin Exp Res 40:717-727.

Saura CA, Parra-Damas A, Enriquez-Barreto L (2015) Gene expression parallels synaptic excitability and plasticity changes in Alzheimer's disease. Front Cell Neurosci 9:318.

Saxena S, Caroni P (2011) Selective neuronal vulnerability in neurodegenerative diseases: from stressor thresholds to degeneration. Neuron 71:35-48.

Schmitz C, Rutten BPF, Pielen A, Schäfer S, Wirths O, Tremp G, Czech C, Blanchard V, Multhaup G, Rezaie P, Korr H, Steinbusch HWM, Pradier L, Bayer TA (2004) Hippocampal neuron loss exceeds amyloid plaque load in a transgenic mouse model of Alzheimer's disease. Am J Pathol 164:1495-1502.

Serrano-Pozo A, Frosch MP, Masliah E, Hyman BT (2011) Neuropathological alterations in Alzheimer disease. Cold Spring Harb Perspect Med 1:a006189.

Shen-Orr SS, Gaujoux R (2013) Computational deconvolution: extracting cell type-specific information from heterogeneous samples. Curr Opin Immunol 25:571-578.

Srinivasan K, Friedman BA, Larson JL, Lauffer BE, Goldstein LD, Appling LL, Borneo J, Poon C, Ho T, Cai F, Steiner P, van der Brug MP, Modrusan Z, Kaminker JS, Hansen DV (2016) Untangling the brain's neuroinflammatory and neurodegenerative transcriptional responses. Nat Commun 7:11295.
Stein TD, Anders NJ, DeCarli C, Chan SL, Mattson MP, Johnson JA (2004) Neutralization of transthyretin reverses the neuroprotective effects of secreted amyloid precursor protein (APP) in APPSW mice resulting in tau phosphorylation and loss of hippocampal neurons: support for the amyloid hypothesis. J Neurosci 24:77077717.

Team RC (2016) R: a language and environment for statistical computing. Vienna: R Foundation for Statistical Computing.

Toker L, Feng M, Pavlidis P (2016) Whose sample is it anyway? Widespread misannotation of samples in transcriptomics studies. F1000Research 5:2103.

Toker L, Mancarci BO, Tripathy S, Pavlidis P (2018) Transcriptomic evidence for alterations in astrocytes and parvalbumin interneurons in subjects with bipolar disorder and schizophrenia. Biol Psychiatry 84:787-796.

Tseveleki V, Rubio R, Vamvakas S-S, White J, Taoufik E, Petit E, Quackenbush J, Probert L (2010) Comparative gene expression analysis in mouse models for multiple sclerosis, Alzheimer's disease and stroke for identifying commonly regulated and diseasespecific gene changes. Genomics 96:82-91.

Webster SJ, Bachstetter AD, Nelson PT, Schmitt FA, Van Eldik LJ (2014) Using mice to model Alzheimer's dementia: an overview of the clinical disease and the preclinical behavioral changes in 10 mouse models. Front Genet 5:88.

Wirz KTS, Keitel S, Swaab DF, Verhaagen J, Bossers K (2014) Early molecular changes in Alzheimer disease: can we catch the disease in its presymptomatic phase? J Alzheimers Dis 38:719-740.

Wood WG, Li L, Müller WE, Eckert GP (2014) Cholesterol as a causative factor in Alzheimer's disease: a debatable hypothesis. $J$ Neurochem 129:559-572.

Wright AL, Zinn R, Hohensinn B, Konen LM, Beynon SB, Tan RP, Clark IA, Abdipranoto A, Vissel B (2013) Neuroinflammation and neuronal loss precede $A \beta$ plaque deposition in the hAPP-J20 mouse model of Alzheimer's disease. PLoS One 8:e59586.

Yang Y, Song W (2013) Molecular links between Alzheimer's disease and diabetes mellitus. Neuroscience 250:140-150.

Zotova E, Holmes C, Johnston D, Neal JW, Nicoll J. a R, Boche D (2011) Microglial alterations in human Alzheimer's disease following $A \beta 42$ immunization. Neuropathol Appl Neurobiol 37:513-524.

Zoubarev A, Hamer KM, Keshav KD, McCarthy EL, Santos JRC, Van Rossum T, McDonald C, Hall A, Wan X, Lim R, Gillis J, Pavlidis P (2012) Gemma: a resource for the reuse, sharing and metaanalysis of expression profiling data. Bioinforma Oxf Engl 28: 2272-2273. 